\title{
SARCOMA DE KAPOSI ENDEMICO EN UN PACIENTE VIH NEGATIVO
}

\author{
José Revilla-López ${ }^{1,2, b}$, Raúl Mendoza-Fabián²,3,a , Andrea Anampa-Guzmán,3,a, Josias Rosales-Zúñigaa,3,a, \\ Joel Chalco-Huamán $2,3, a$
}

\begin{abstract}
RESUMEN
El sarcoma de Kaposi (SK) es un cáncer angioproliferativo inflamatorio multifocal asociado a herpes virus 8 (VHH-8). Se han descrito cuatro variantes clínico-epidemiológicas: clásico, endémico, iatrogénico y epidémico o asociado a VIH. Clínicamente puede ser indolente o agresivo, afecta principalmente áreas mucocutáneas con eventual compromiso visceral y de ganglios linfáticos. Se presenta frecuentemente y de forma más agresiva en la población VIH positiva. Presentamos un caso de un paciente varón de 27 años VIH negativo con lesión tumoral sangrante en el anillo de Waldeyer, múltiples adenopatías y lesiones exofíticas en pie que remiten con quimioterapia de emergencia basada en antraciclinas. EI SK VIH negativo es una condición poco frecuente. Es importante tener en cuenta al Perú como región endémica para el VHH-8. La afectación oral del SK es una manifestación rara y de mal pronóstico, sin embargo, el factor VIH negativo podría conferirle un buen pronóstico.
\end{abstract}

Palabras clave: Sarcoma de Kaposi; Neoplasias; VIH (fuente: DeCS BIREME).

\section{ENDEMIC KAPOSI'S SARCOMA IN A HIV-NEGATIVE PATIENT}

\begin{abstract}
Kaposi's sarcoma (KS) is a multicentric, inflammatory angioproliferative cancer associated with herpes virus 8 (HHV-8). It has four clinico-epidemiological types: classic, endemic, iatrogenic and epidemic, or HIV associated. Clinically it may be indolent or aggressive, mainly affecting mucocutaneous areas with eventual visceral and lymph node involvement. It is often present and in a more aggressive form in the HIV-positive population. We report a case of a 27 -year-old HIVnegative male patient with a bleeding tumoral lesion in the Waldeyer ring, multiple lymphadenopathies and exophytic foot lesions that remit with anthracycline-based emergency chemotherapy. HIV-negative KS is a rare condition. It is important to consider Peru as an endemic region for HHV-8. The oral involvement of KS is a rare manifestation and of poor prognosis, however, the HIV-negative factor could confer a good prognosis.
\end{abstract}

Key words: Sarcoma, Kaposi; Neoplasms; HIV (source: MeSH NLM).

\section{INTRODUCCIÓN}

En el año 1872, Moritz Kaposi describió una lesión de piel como "sarcoma múltiple pigmentado idiopático" (1). Hoy en día se sabe que el sarcoma de Kaposi (SK) es una enfermedad angioproliferativa inflamatoria multifocal asociada a virus herpes humano-8 $(\mathrm{VHH}-8)^{(2)}$. Se clasifica en cuatro variantes clínico-epidemiológicas: clásico, endémico, iatrogénico y epidémico o asociado a VIH. Con el aumento de la incidencia del VIH SIDA la forma epidémica o asociada a VIH actualmente es la más frecuente.

Clínicamente, se han observado múltiples manifestaciones que afectan principalmente áreas mucocutáneas, produciendo típicas lesiones en nódulos, pápulas y/o parches oscuros, con eventual compromiso visceral y de ganglios linfáticos (3). La evolución clínica del SK puede ser indolente o agresiva. El diagnostico de SK está basado en las

\footnotetext{
Hospital Nacional Daniel Alcides Carrión. Lima, Perú.

Facultad de Medicina, Universidad Nacional Mayor de San Marcos. Lima, Perú.

Sociedad Científica de San Fernando.

a Estudiante de Medicina; ${ }^{\mathrm{b}}$ médico oncólogo

Este reporte ha sido presentado en el XXVIII Congreso Científico Nacional SOCIMEP Arequipa, Perú 2014 y en el XXIX Congreso Científico

Internacional FELSOCEM Córdoba, Argentina 2014.

Recibido: 07-04-15 Aprobado: 05-08-15
}

Citar como: Revilla-López J, Mendoza-Fabián R, Anampa-Guzmán A, Rosales-Zúñiga J, Chalco-Huamán J. Sarcoma de Kaposi endemico en un paciente VIH negativo. Rev Peru Med Exp Salud Publica. 2015;32(4):808-12. 

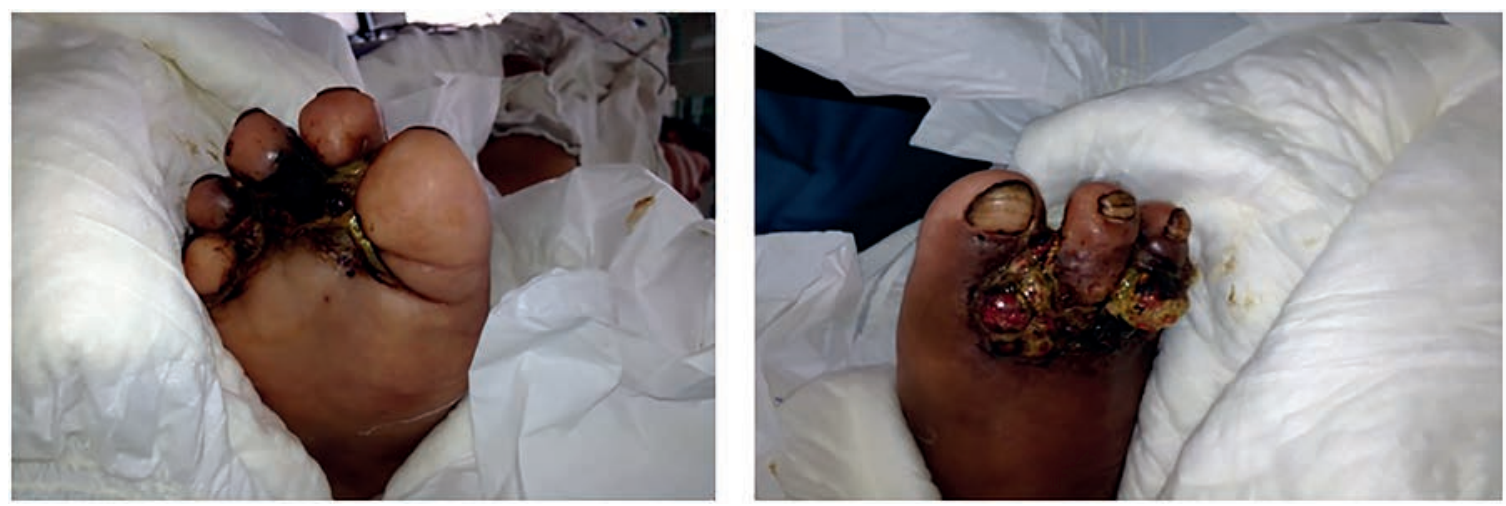

Figura 1. Lesiones exofíticas granulomatosas rojo violáceas necróticas malolientes de $4 \times 3 \mathrm{~cm}$, en planta y dorso del pie derecho entre el $2 .^{\circ}$ y $4 .^{\circ}$ dedo

características histológicas e inmunohistoquímica para la identificación de antígenos ${ }^{(4)}$.

Presentamos el caso infrecuente de un paciente de 27 años, VIH negativo, con SK endémico visceral agresivo que remite con quimioterapia de emergencia basado en antraciclinas.

\section{REPORTE DEL CASO}

Paciente varón de 27 años de edad, natural y procedente de Huánuco, soltero, agricultor, sin antecedentes de importancia. Refiere un tiempo de enfermedad de siete meses que inicia con adenopatías inguinales bilaterales de crecimiento lento progresivo. Dos meses después presenta adenopatías en cuello y axilas, no dolorosas, induradas y de crecimiento progresivo; además de una lesión granulomatosa en el pie derecho. En el hospital recibe tratamiento analgésico y curación tópica, sin embargo, la lesión progresa a múltiples lesiones verrugosas exofíticas en la planta y dorso del pie derecho. Dos meses después se añaden cambios en la voz, disfagia y palpación de masa en cavidad oral. Luego de dos meses presenta tos sanguinolenta, palidez, equimosis de globo ocular y en cara anterolateral del muslo izquierdo y aumento de volumen en miembros inferiores. Finalmente, dos días antes del ingreso presenta lesión bucal sangrante, malestar general e hiporexia por lo que acude al servicio de emergencia.

Al ingreso, los signos vitales era normales, hemodinámicamente estable, pero con palidez moderada y múltiples lesiones exofíticas granulomatosas interdigitales en pie derecho en dorso y planta, con aumento de volumen, signo de flogosis y secreción fétida (Figura 1). En el anillo de Waldeyer, en la cavidad oral, se observó una lesión tumoral sangrante que obstruía la vía aérea en un $40 \%$, además de múltiples adenomegalias cervicales, retroauriculares, claviculares y axilares, la mayor de ellas de $3 \mathrm{~cm}$ y el resto de $1 \mathrm{~cm}$. La región inguinal presentaba adenopatías induradas de $5 \mathrm{~cm}$, adheridas a planos profundos, no dolorosas, que generaban edema.

En los exámenes auxiliares de ingreso se encontró anemia grado $3(\mathrm{Hb}: 6,1 \mathrm{~g} / \mathrm{dL})$ y plaquetopenia grado $2\left(70000 \mathrm{~mm}^{3}\right)$, además de hipoalbuminemia grado 1 $(2,9 \mathrm{~g} / \mathrm{dL})$ y perfil de coagulación con INR (International normalized ratio) incrementado.

Se plantearon los siguiente diagnósticos diferenciales: linfoma no Hodking, sarcoma de Kaposi, melanoma, y carcinoma epidermoide. Dentro del plan de trabajo se consideró: exámenes de control, test de Coombs, deshidrogenasa láctica sérica, estudio de médula ósea, biopsia de ganglio y lesión de pie, examen serológico viral (VIH, VDRL, HTLV-1) con test de ELISA y estudios de extensión de enfermedad con tomografías.

El paciente tuvo una evolución estacionaria hasta el quinto día que presento sangrado masivo por boca, motivo por el cual ingresa a $\mathrm{UCl}$ (unidad de cuidados intensivos). Presento shock hipovolémico con insuficiencia respiratoria y anemia grado 4 por lo que se decide la ventilación mecánica y trasfusión sanguínea. La lesión tumoral oral obstruía el $80 \%$ de la vía aérea y sangraba masivamente. Los resultados de los estudios fueron: bicitopenia e incremento de la bilirrubina a predominio indirecto. Aspirado y biopsia de medula ósea: reactivo, sin células neoplásicas. Test de Coombs: negativo. Antígeno de superficie: no reactivo. VDRL, RPS, VIH y HTLV-1: no reactivo. Acute Physiology and Chronic Health Evaluation (APACHE): 7. Eastern Cooperative Oncologic Group (ECOG): 3.

Se consideró el caso como una emergencia oncológica por lo que se decide iniciar quimioterapia de emergencia con un esquema basado en antraciclinas (ciclofosfamida, vincristina, doxorrubicina, prednisona) 


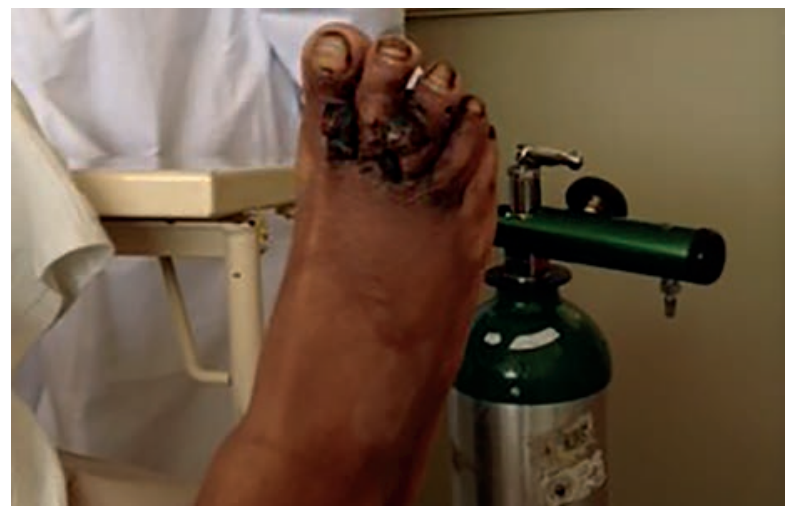

Figura 2. Lesiones exofíticas granulomatosas en resolución. Evolución posterior a la quimioterapia con esquema basado en antraciclinas

según las características clínicas de la enfermedad, con alta sospecha de linfoma no Hodking y sarcoma de Kaposi. Se repite prueba de $\mathrm{VIH}$, la cual resulta negativa.

El paciente recibió el esquema basado en antraciclinas por un ciclo, con evolución favorable. Las lesiones exofíticas granulomatosas en pie y las adenopatías axilares, inguinales y cervicales disminuyeron en número y tamaño. Los niveles de laboratorio mejoraron (Figura 2).

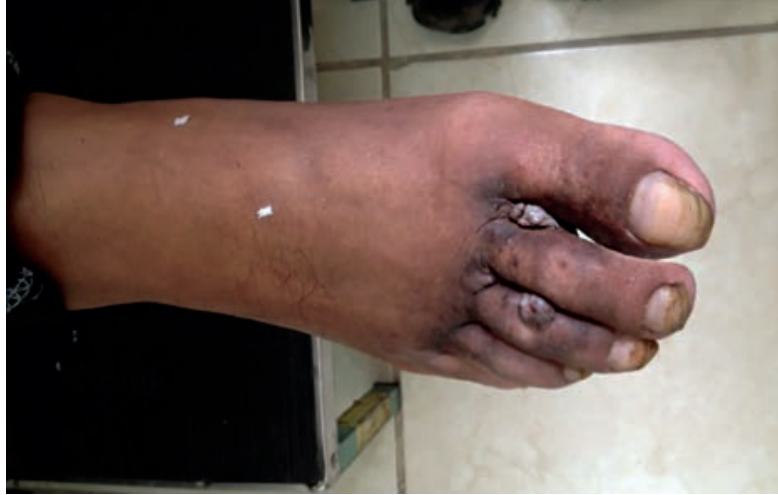

Figura 4. Lesiones exofíticas granulomatosas en resolución, durante el alta hospitalaria

El servicio de Anatomía Patológica envía el resultado de la biopsia de ganglio el cual fue compatible con sarcoma de Kaposi, HHV-8 (+), CD34: positivo, CD 31: positivo (Figura 3).

Se confirmado el diagnostico de un varón de 27 años con sarcoma de Kaposi visceral VIH negativo con factores pronóstico desfavorables, el tratamiento fue cambió a paquitaxel, recibió siete ciclos con evolución favorable (Figura 4); se recomendó un octavo y noveno ciclo, pero el paciente abandonó el tratamiento para volver a Huánuco, en donde se perdió el contacto.

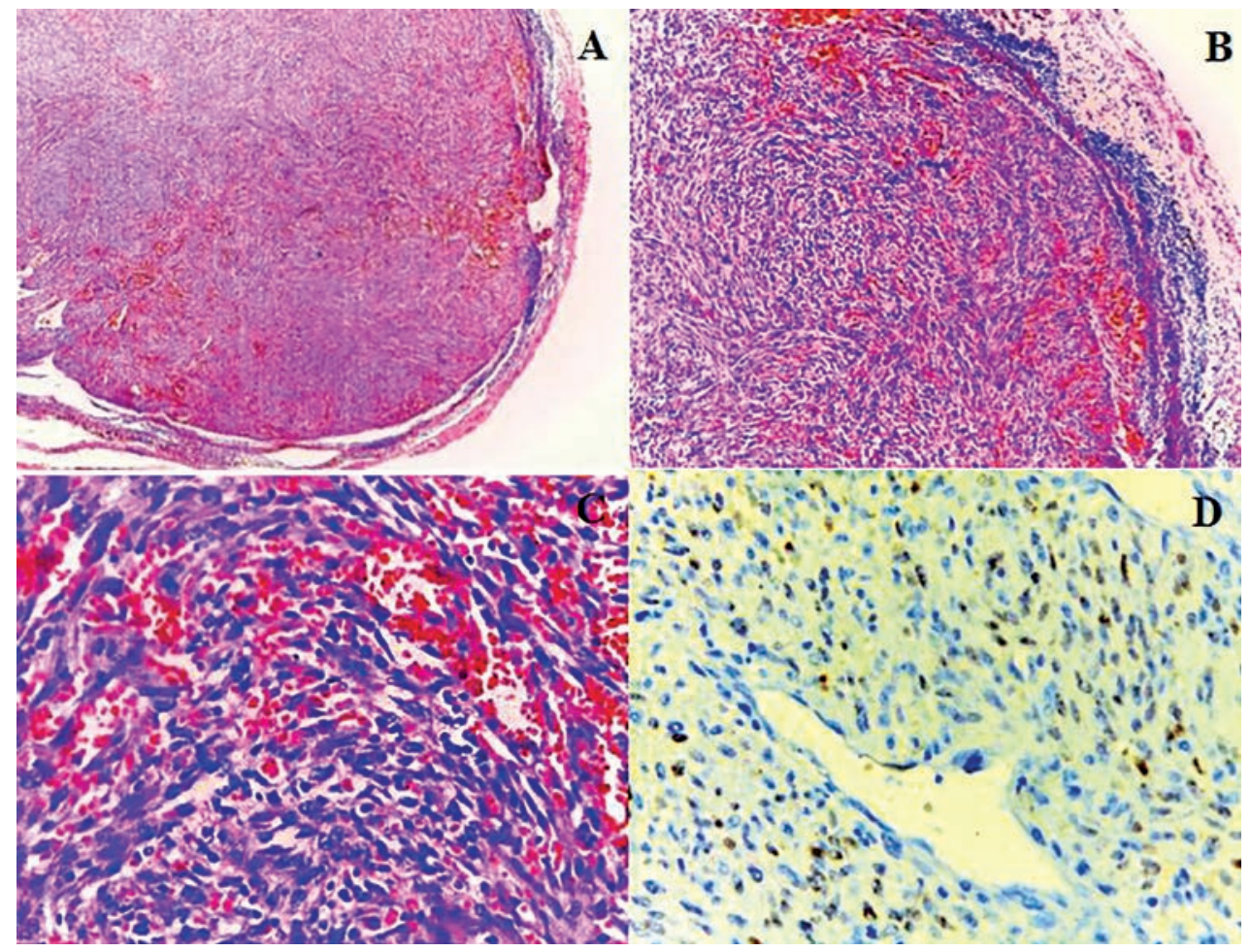

Figura 3. Biopsia de ganglio. Se observa proliferación metastásica fusocelular con hendiduras vasculares, tinción: hematoxilinaeosina, aumento 4X (A), 10X (B), 40X (C) y la inmunohistoquímica con el herpesvirus tipo 8 positivo, aumento 10X (D) 


\section{DISCUSIÓN}

El VHH-8 es actualmente considerado como el agente etiológico del linfoma primario de cavidades, la enfermedad de Castleman multicéntrica y el sarcoma de Kaposi (SK) ${ }^{(5)}$.

El VHH-8 está distribuido mundialmente, pero su prevalencia varía según el área geográfica y concuerda con la prevalencia de SK registrada (6); en EE.UU., norte de Europa y Asia se han registrado bajas seroprevalencias $(<10 \%)$; en la región mediterránea se reporta una mediana seroprevalencia (10-30\%) y en el centro y sur de África una alta seroprevalencia $(>50 \%)^{(7)}$. En América del Sur se ha encontrado una alta seroprevalencia en poblaciones indígenas de Brasil, Ecuador, Colombia y Guayana Francesa ${ }^{(8)}$. Un estudio en donadores de sangre en Perú encontró una seroprevalencia mayor del $50 \%$, por lo que se considera una zona con alta endemicidad de $\mathrm{VHH}-8{ }^{(9)}$. Esta información es relevante ya que el paciente es natural y procedente de una zona altoandina de Perú.

La variante endémica del SK se describe en personas de mediana edad (25-50 años) VIH negativos, provenientes de zonas con alta endemicidad de HHV-8. Es por ello que, considerando las características clínicas y epidemiológicas del paciente, se tratarían de un SK de la variante endémica ${ }^{(3)}$.

Con el advenimiento del VIH-SIDA la variante epidémica o asociada al VIH del SK actualmente es la más frecuente, por otro lado, el SK en personas VIH (-) es una condición poco usual; sin embargo, existen reportes que afirman mayor frecuencia en poblaciones indígenas de América del Sur (andinas, amazónicas y quechuas) ${ }^{(10)}$. Además, es importante resaltar que los casos de SK VIH (-) en poblaciones indígenas pueden estar subdiagnosticados, debido a los bajos recursos económicos y poca accesibilidad a centros de salud.
En la evolución del paciente se presentó una lesión sangrante en el anillo de Waldeyer, esta lesión le producía insuficiencia respiratoria y shock hipovolémico por el sangrado. Las lesiones orales en pacientes con SK son frecuentes en pacientes $\mathrm{VIH}$ positivos, y representan el $70 \%$ de los casos en piel y vísceras, además, están asociadas a un mal pronóstico (11). Mientras que en pacientes VIH negativos, como el paciente de este estudio, las lesiones orales son poco usuales ${ }^{(12)}$. Probablemente la respuesta favorable al tratamiento sea por el factor $\mathrm{VIH}$ negativo.

El tratamiento del SK es individualizado y, depende de factores como la variante clínica, el número y localización de las lesiones, el estado inmune del paciente, y si existe afectación visceral y/o ganglionar (4). La quimioterapia es usada en las formas rápidamente progresivas o con afectación sistémica; en la actualidad la FDA (Food and Drug Administration) ha aprobado tres agentes quimioterapéuticos: doxorrubicina liposomal pegilada, daunorubicina liposomal y paclitaxel, siendo la doxorrubicina liposomal el agente más usado y mejor tolerado (13). La terapia combinada de bleomicina, vincristina y doxorrubicina estándar ha sido remplazada por su baja eficacia y elevada toxicidad; sin embargo, aún puede ser utilizada cuando es inviable la prescripción de la doxorrubicina liposomal (14), esto puede explicar por qué el esquema basado en antraciclinas en el caso presentado tuvo una respuesta favorable e hizo que las lesiones remitieran, similar a lo expuesto, existe un reporte de caso de SK tratado con un esquema similar con resultados favorables ${ }^{(15)}$, reafirmando la utilidad de la terapia con esquema basado en antraciclinas en pacientes $\mathrm{SK} \mathrm{VIH}(-)$.

Contribuciones de autoría: JMF y AAG han participado en la concepción y diseño del artículo. JMF, AAG, JRZ y JCH contribuyeron con la redacción del artículo, JRL participó en la revisión crítica del artículo y la aprobación de su versión final.

Fuentes de financiamiento: autofinanciado

Conflictos de interés: los autores declaran no tener conflictos de interés.

\section{REFERENCIAS BIBLIOGRÁFICAS}

1. Braun M. Classics in Oncology. Idiopathic multiple pigmented sarcoma of the skin by Kaposi. CA Cancer J Clin 1982;32(6):340-7.

2. Chang Y, Cesarman E, Pessin MS, Lee F, Culpepper J, Knowles DM, et al. Identification of herpesviruslike DNA sequences in AIDSassociated Kaposi's sarcoma. Science. 1994;266(5192):1865-9.

3. Szajerka T, Jablecki J. Kaposi’s sarcoma revisited. AIDS Rev 2007;9(4):230-6.
4. IARC. Biological Agents. Kaposi Sarcoma Herpesvirus. IARC Monogr Eval Carcinog Risks Hum. 2012; 100B:169-214.

5. Kaplan LD. Human herpesvirus-8: Kaposi sarcoma, multicentric Castleman disease, and primary effusion lymphoma. Hematology Am Soc Hematol Educ Program. 2013;2013:103-8. doi: 10.1182/asheducation-2013.1.103.

6. Rohner E, Wyss N, Trelle S, Mbulaiteye S, Egger M, Novak U et al. HHV-8 seroprevalence: a global view. Syst Rev. 2014;3:11. doi: 10.1186/2046-4053-311.

7. Dukers NH, Rezza G. Human herpesvirus 8 epidemiology: what we do and do not know. AIDS. 2003;17:1717-30.

8. Souza VA, Salzano FM, Petzl-Erler ML, Nascimento MC, Mayaud P, Borges JD, et al. Variations in Human Herpesvirus Type 8 Seroprevalence in Native Americans, South America. 
Emerg Infect Dis. 2010;16(6):1003-6. doi: 10.3201/eid1606.090961.

9. Mohanna S, Portillo JA, Carriquiry G, Vidal J, Ferrufino JC, Sanchez J, et al. Human herpesvirus- 8 in Peruvian blood donors: A population with hyperendemic disease? Clin Infect Dis. 2007;44(4):558-61.

10. Mohanna S, Maco V, Bravo F, Gotuzzo E. Epidemiology and clinical characteristics of classic Kaposi's sarcoma, seroprevalence, and variants of human herpesvirus 8 in South America: A critical review of an old disease. Int J Infect Dis. 2005;9(5):239-50.
11. Pantanowitz L, Khammissa RA, Lemmer J, Feller L. Oral HIVassociated Kaposi sarcoma. J Oral Pathol Med. 2013;42(3):201-7. doi: 10.1111/j.1600-0714.2012.01180.x.

12. Fatahzadeh M, Schwartz RA. Oral Kaposi's sarcoma: a review and update. Int JDermatol.2013;52(6):666-72. doi: 10.1111/j.1365-4632.2012.05758.x.

13. Wood BR, Sax PE. Current approaches to treating kaposi sarcoma. AIDS Clin Care. 2011;2011(613):1.

14. Northfelt DW, Dezube BJ, Thommes JA, Miller BJ, Fischl MA, FriedmanKien A, et al. Pegylated-liposomal doxorubicin versus doxorubicin, bleomycin and vincristine in the treatment of AIDS-related Kaposi's sarcoma: Results of a randomized phase III clinical trial. J Clin Oncol. 1998;16(7):2445-51.

15. Sanz M, Feinsilber D, Corbella C, Schröh R, Hassan M. Tumores raros de difícil diagnóstico inicial. Dermatol Argent. 2009;15(4):272-7.

Correspondencia: Andrea Carolina
Anampa Guzmán
Dirección: Av. Villaverde 308 Urb. San
Amadeo de Garagay.Lima, Perú.
Teléfono: (+511)998455065
Correo electrónico:andreaangu11@hotmail.com

\section{http://facebook.com/rpmesp}

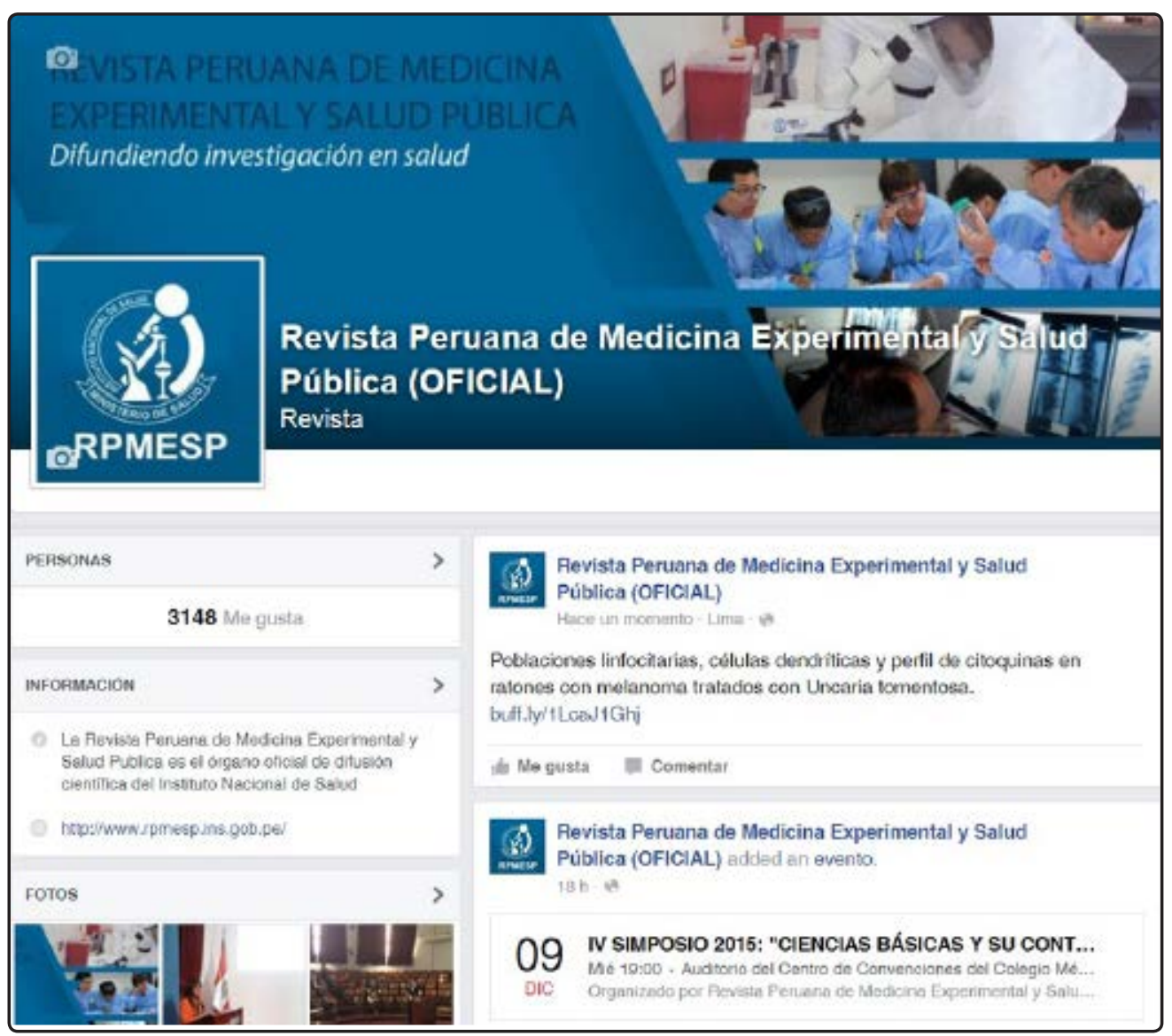

